\title{
Immunotherapy for mesothelioma: a critical review of current clinical trials and future perspectives
}

\author{
Steven G. Gray ${ }^{1}$, Luciano Mutti ${ }^{2}$ \\ ${ }^{1}$ Thoracic Oncology Research Group, Trinity St. James's Cancer Institute, Trinity Translational Medicine Institute, Trinity Centre for Health \\ Sciences, St. James's Hospital, Dublin, Ireland; ${ }^{2}$ Center for Biotechnology, Sbarro Institute for Cancer Research and Molecular Medicine, College of \\ Science and Technology, Temple University, Philadelphia, PA, USA \\ Contributions: (I) Conception and design: All authors; (II) Administrative support: All authors; (III) Provision of study materials or patients: None; \\ (IV) Collection and assembly of data: All authors; (V) Data analysis and interpretation: All authors; (VI) Manuscript writing: All authors; (VII) Final \\ approval of manuscript: All authors. \\ Correspondence to: Steven G. Gray. Thoracic Oncology Research Group, Trinity Translational Medical Institute, Central Pathlogy Laboratory, St. James's \\ Hospital D08RX0X, Dublin, Ireland. Email: sgray@stjames.ie; Professor Luciano Mutti. Center for Biotechnology, Sbarro Institute for Cancer Research \\ and Molecular Medicine, College of Science and Technology, Temple University, Philadelphia, PA 19122, USA. Email: chairman@gime.it.
}

\begin{abstract}
At the clinical level the role of immunotherapy in cancer is currently at a pivotal point. Therapies such as checkpoint inhibitors are being approved at many levels in cancers such as non-small cell lung cancer (NSCLC). Mesothelioma is a rare orphan disease associated with prior exposure to asbestos, with a dismal prognosis. Various clinical trials for checkpoint inhibitors have been conducted in this rare disease, and suggest that such therapies may play a role as a treatment option for a proportion of patients with this cancer. Most recently approved as a salvage therapy in mesothelioma was granted in Japan, regulatory approval for their use in the clinic elsewhere lags. In this article we review the current pertinent clinical trials of immunotherapies in malignant mesothelioma, discuss the current issues that may affect the clinical outcomes of such therapies and further evaluate potential candidate new avenues that may become future targets for immunotherapy in this cancer.
\end{abstract}

Keywords: Checkpoint inhibitors; immunotherapy; co-stimulatory; biomarkers; vaccine; dendritic cell; mesothelioma

Submitted Aug 23, 2019. Accepted for publication Nov 14, 2019.

doi: $10.21037 /$ tlcr.2019.11.23

View this article at: http://dx.doi.org/10.21037/tlcr.2019.11.23

\section{Introduction}

Malignant pleural mesothelioma (MPM) is an aggressive inflammatory cancer associated with prior exposure to asbestos. There is a myth that MPM is disappearing based on the notion that as the causative agent asbestos has been banned in the western world, this should cause a corresponding drop in MPM from the 1990's onwards. However, data from the US shows that the rate of MPM in males has remained constant from 1994, while the rate of MPM in females has remained unchanged for decades (1). While industrial use of asbestos has declined in industrialized nations, asbestos is still being exported to developing nations and in developed nations environmental exposure is widespread due to previous industrial use, its difficulty to remove, and because natural deposits are being disturbed by human activities or housing is built near to these deposits (2-4).

Untreated, MPM has a median survival time of 6 months, and most patients die within 24 months of diagnosis. The current standard of care (SoC) (combination of pemetrexed and cisplatin chemotherapy) (5) is non-curative and results in a response rate of $\sim 40 \%$ (6), and there is no standard second line therapy once treatment fails. Currently Bevacizumab, an anti-VEGF therapy is the first drug which when added to the SoC enhances OS in the first line setting $(7,8)$. However, 
this therapeutic combination is not currently widely used as it has yet to be given FDA approval, issues with cost and lack of reimbursement in many countries. The only other recent FDA approval for mesothelioma has been the approval of the NovoTTF TM 100 L System (9). This system is a device which uses alternating electric fields at specific frequencies and intensities to selectively disrupt mitosis in cancerous cells (10) and currently FDA approved for the treatment of glioblastoma (11). In the STELLAR mesothelioma trial (ClinicalTrials.gov NCT02397928) this system was found to improve the median OS in patients enrolled to 18.2 months (12). Of 80 enrolled patients with unresectable mesothelioma, the median overall survival was 21.2 months for patients with epithelioid MPM ( $\mathrm{n}=53)$ and 12.1 months for patients with non-epithelioid MPM (n=21). Around 62 percent of participants who used the device concurrently with pemetrexed and platinum-based chemotherapy were alive at one year, although concerns exist as to whether potential inherent biases and lack of sufficient controls can allow for a true interpretation of the therapeutic value of this system in mesothelioma.

In 1909, Paul Ehrlich postulated that the immune system had the ability to suppress the majority of carcinomas and thus play an important role in the body's defenses against tumor development (13). Immunotherapy is thus a situation where the patient's own immune system is exploited in order to eliminate tumor cells, and has become one of the most prominent new cancer treatment options in the last decade (13). Immunotherapy can be classified into either active immunotherapy which aims to stimulate the patient's immune system, typically through vaccination, or passive immunotherapy, in which immune effectors are isolated in vitro before applying to the patient (14). It took many decades of ground-breaking work to demonstrate that cancer immunotherapy was a viable treatment option (15) and resulted in the Nobel Prize being awarded to James Allison at the University of Texas MD Anderson Cancer Center in Houston and Tasuku Honjo at Kyoto University in Japan for their efforts in this field (16).

The paucity of treatment options available to patients following failure of first-line treatment has provided a unique window of opportunity within the last five years to test immunotherapies in mesothelioma. In this review we discuss the current clinical trials of immunotherapies, the issues associated with clinical responses or lack thereof, and examine some of the alternative immunotherapy options currently within the clinical development pipeline which could potentially be translated into mesothelioma clinical trials moving forwards.

\section{Immunotherapy in MPM in the historical setting}

Early studies on immunotherapy in mesothelioma have been tried for over 25 years (17), beginning with various trials using interferons to attempt to induce tumor directed mobilization of macrophages (18-20). These trials generally had median survival rates of approximately 8-12 months. In one of these trials those patients who had an objective response (OR) had a significantly longer median time to progression (21 months) and survival time (25 months) than non-responders ( 3 and 8 months, respectively) (19). Moreover, a subsequent Phase II study involving intrapleural infusion of interferon- $\gamma$ and activated macrophages observed a median survival for all treated 29.2 months (21). More recently Phase I trials involving intra-pleural adenoviral mediated interferon therapy have been conducted (22-24). In the most recent of these patients with unresectable MPM received two intra-pleural doses of an adenoviral vector containing the human IFN $\alpha 2 \mathrm{~b}$ gene (Ad.IFN) concomitant with a 14-day course of celecoxib followed by either first-line pemetrexed-based chemotherapy $(n=18)$ or second-line chemotherapy with pemetrexed or gemcitabine $(n=22)$. Following completion of the study, median overall survival in the first-line cohort was 12.5 months, whereas in the second-line chemotherapy cohort it was 21.5 months, with $32 \%$ of patients alive at 2 years (22).

Another early potential immunotherapy target identified in mesothelioma was granulocyte-macrophage colonystimulating factor (GM_CSF) (21). Several initial trials involving infusions of GM-CSF (25-27) and either had few or no responses $(26,27)$ or had a poor overall survival (median survival of 7 months), coupled with high toxicity (25). A small clinical trial ( $\mathrm{n}=22$ patients) was conducted involving a vaccination strategy comprising autologous mesothelioma tumor cell lysate combined with GM-CSF was conducted. The trial was found to be safe, and induced tumor specific immunity in $32 \%$ of patients, but saw only stable disease ad no tumor ORs (28). More recently, tumor derived GM-CSF was shown to actually promote immunosuppression in mesothelioma suggesting that actually targeting this molecule may be more effective in augmenting immunotherapy in MPM (29).

Various other early trials have been conducting for example using Interleukin-2 and TNF- $\alpha$. Most of these were ineffective and suffered from various problems such 
Table 1 Neoadjuvant Checkpoint Inhibitor Trials in malignant pleural mesothelioma (MPM) discussed in this article

\begin{tabular}{lccccc}
\hline Agent & Phase & Identifier & Target & Number of patients & Status \\
\hline Nivolumab; ipilimumab & II/III & NCT03918252 & Anti-PD-1; & 30 & Not yet recruiting \\
& & & Anti-CTLA4 & & Recruiting \\
Atezolizumab & I & NCT03228537 & Anti-PD-L1 & 28 & (31) \\
Pembrolizumab & I & NCT02707666 & Anti-PD-1 & 15 & Recruiting \\
Pembrolizumab & $\mathrm{I}$ & NCT02959463 & Anti-PD-1 & 24 & Recruiting \\
Tremelimumab; durvalumab & $\mathrm{II}$ & NCT02592551 & Anti-CTLA4; & 20 & Recruiting
\end{tabular}

as lack of scalability and logistical issues $(17,30)$. However, a new Phase III study - (INFINITE - NCT03710876) is currently recruiting for a trial involving intra-pleural administration of TR002 an adenovirus-delivered Interferon Alpha-2b (rAd-IFN) and examining its efficacy and safety in combination with celecoxib and gemcitabine in patients with mesothelioma.

\section{Checkpoint inhibitor immunotherapy within the neo-adjuvant setting}

Although not SoC, there is compelling evidence that a select subgroup of mesothelioma patients benefits from a surgerybased multimodal approach, particularly if they have an epithelioid histological subtype, lower-volume disease, and/or minimal to no nodal involvement (31). As it is not possible to achieve a microscopically complete resection with mesothelioma, there appears to be no role for surgery alone. As such patients who have surgically resectable disease often undergo an aggressive multi-modality therapy for which the optimal combination therapy has yet to be identified $(1,32,33)$. A National Cancer InstituteInternational Association for the Study of Lung CancerMesothelioma Applied Research Foundation Mesothelioma Clinical Trials Planning Meeting was held in 2017 which set up a taskforce to explore this situation, and new consensus reports have just been published $(34,35)$.

Neoadjuvant immunotherapy prior to surgery has been mooted as an advantageous prospect in the management of solid tumors as they enhance T-cell activation the moment antigen is encountered (36), and encouraging findings from early-phase clinical trials in various cancers support this notion (37).

However, clinical trials involving neo-adjuvant immunotherapy in mesothelioma are not yet mature.
Several have been initiated (as presented in Table 1) and will be briefly discussed in the following sections.

NCT02707666 is a single-institution, Phase I "window of opportunity" study of Pembrolizumab given prior to surgery in $(\mathrm{n}=15)$ patients with resectable MPM (38). All patients will undergo a pre-treatment PET/CT scan for clinical staging and a VATS procedure to acquire pretreatment tissue. Three cycles of pembrolizumab are to be administered (200 mg intravenously every 21 days). A PET/CT scan will then be repeated to assess response to pembrolizumab and then surgical resection will be performed via an extended/pleurectomy decortication at least 4 weeks after the third dose of pembrolizumab. Standard adjuvant chemotherapy consisting of cisplatin and pemetrexed for 4 cycles (every 21 days) will be given starting about 6-8 weeks following surgery, after a new baseline CT scan is obtained. Restaging CT scans will be obtained to assess response after every two cycles of chemotherapy. After the completion of standard chemotherapy, optional adjuvant treatment with pembrolizumab will be given to eligible patients for 1-year post-surgery.

The primary objective is to assess an increase in interferon- $\gamma$, measured via a gene expression profile (GEP), comparing matched pre- and post-treatment samples (IFN-G GEP response), and to identify additional candidate biomarkers that may predict benefit or constitutive resistance to pembrolizumab (38). This trial was expected to complete in September 2018.

Another Phase I neoadjuvant trial involving Pembrolizumab is NCT02959463, which aims to study the side effects and best way to give pembrolizumab after radiation therapy in treating patients with pleural malignant mesothelioma. In this trial patients are assigned to 1 of 2 cohorts. The first cohort undergoes hemi-thoracic radiation therapy, and following radiation therapy, patients receive 
Table 2 Front-line checkpoint inhibitor trials in MPM discussed in this article

\begin{tabular}{|c|c|c|c|c|c|c|}
\hline Agent & Phase & Identifier & Target & Number of patients & Status & Reference \\
\hline Nivolumab; ipilimumab & III & NCT02899299 & $\begin{array}{l}\text { Anti-PD-1; } \\
\text { Anti-CTLA4 }\end{array}$ & $\mathrm{n}=600$ & $\begin{array}{l}\text { Active, not } \\
\text { recruiting }\end{array}$ & (41) \\
\hline
\end{tabular}

*, considered to be part front-line as patients could enrol, if they had refused a first line platinum-based chemotherapy. MPM, malignant pleural mesothelioma.

pembrolizumab intravenously and repeated every 3 weeks for up to 2 years in the absence of disease progression or unacceptable toxicity. In the second cohort, the patients undergo palliative radiation therapy over $1-3$ weeks to only the region of palliation and then receive intravenous pembrolizumab similar to cohort 1 . After completion of study treatment, patients will be followed up at 30 days, every 6 weeks for 48 weeks, then every 12 weeks for up to 5 years. The primary objective will be to determine the safety and tolerability of pembrolizumab administered after radiation therapy in patients with MPM who have not undergone extra-pleural pneumonectomy. The secondary objectives are progression-free survival (PFS) and OS.

A phase I trial of atezolizumab combined as multimodality with $1^{\text {st }}$-line cisplatin/pemetrexed combined with surgery and/or radiation therapy in stage I-III pleural malignant mesothelioma is currently recruiting (31). In this single-group feasibility trial $(\mathrm{n}=28)$ patients will first undergo neoadjuvant immunotherapy combined with firstline chemotherapy, then surgical resection (extra-pleural pneumonectomy EPP, or pleurectomy with decortication P/ D), followed finally by radiotherapy for those patients who underwent EPP. Maintenance atezolizumab will then be given for up to 1 year in the absence of disease progression or unexpected toxicity. The primary objectives are PFS, OS and mRECIST PFS (28). The estimated completion study date for this trial is June 1, 2020.

Two Phase II clinical trials are currently running involving combined neo-adjuvant checkpoint inhibitors. The first (NCT02592551), involves a study examining whether durvalumab or combination therapy with durvalumab plus tremelimumab is associated with favorable alterations of the intra-tumoral immunologic environment in mesothelioma patients undergoing surgery and is estimated to complete in December, 2019. In this small study of ( $\mathrm{n}=20)$ patients; $\mathrm{n}=4$ will be untreated (control); $\mathrm{n}=8$ will received one intravenous dose of durvalumab alone; and $\mathrm{n}=8$ will receive one intravenous dose of durvalumab plus tremelimumab prior to surgery. The primary outcome measures in this study will be to assess the intra-tumoral $\mathrm{CD}+/$ Treg ratio; the percentage of inducible T-cell costimulator (ICOS) + CD4 T cells; and the tumor expression levels of PD-L1. The second trial (NCT03918252) will examine the safety and feasibility of neoadjuvant nivolumab +/- ipilimumab in resectable MPM. In this study of $\mathrm{n}=30$ patients, 15 will receive three doses of preoperative Nivolumab, while the other 15 will receive a single dose of ipilimumab along with three doses of Nivolumab prior to surgery. The primary outcome is safety and feasibility. Secondary outcomes include pathological and radiological responses and toxicity. This phase II/III trial is currently not yet recruiting, but has an estimated completion time of June 2026.

\section{Checkpoint inhibitor Immunotherapy within the front-line setting}

The data as regards checkpoint inhibitor immunotherapy in the front-line setting is emerging and the main clinical trials which are completed or are ongoing are summarized in (Table 2) and discussed below.

\section{Completed}

The NIBIT-Meso-1 trial can be considered to have some elements of immunotherapy within the frontline setting, as one criterion for patient inclusion was if a patient had refused a first line platinum-based chemotherapy. The results of this trial were published in 2018, and as other patients enrolled in this trial had relapsed after prior 
first-line chemotherapy are discussed in more detail in a subsequent section.

In 2018, the final results of a single-arm, phase 2 trial (DREAM) designed to determine the activity, safety and tolerability of durvalumab, cisplatin and pemetrexed as first line therapy in MPM were presented in abstract form (40). This trial was a non-randomized trial, and $n=54$ patients received durvalumab $1,125 \mathrm{mg}$, cisplatin $75 \mathrm{mg} / \mathrm{m}^{2}$, and pemetrexed $500 \mathrm{mg} / \mathrm{m}^{2}$ all given intravenously on day 1 , and repeated every 3 weeks for a maximum of 6 cycles, followed by durvalumab $1,125 \mathrm{mg}$ every 3 weeks until progression, unacceptable toxicity, or a maximum total of 12 months (40). The primary outcome to be measured was Progression free survival at 6 months (PFS6) according to mRECIST for MPM, which at the end of the trial was determined to be median PFS 6.9 months (40). While the data remains immature, it has been reported that 1-year OS was $65 \%$ at a median follow-up of 14.4 months (42). While this suggests suggests that a triplet regimen of cisplatin-pemetrexed plus durvalumab shows promising responses, and warrants extension into a Phase III clinical trial, it should be noted that five deaths were recorded on this trial and careful selection of patients may thus be required (42).

\section{Ongoing}

Phase III clinical trials of checkpoint inhibitors in the firstline setting have been initiated, and are currently ongoing. The first (Checkmate743) is a phase 3, randomized, openlabel trial of Nivolumab in combination with Ipilimumab versus Pemetrexed with Cisplatin or Carboplatin as first line therapy in unresectable mesothelioma (41). In this trial patients will be randomized 1:1 to receive Nivolumab/ Ipilimumab or pemetrexed and cisplatin/carboplatin. Primary endpoints are OS and PFS. Secondary endpoints are objective response rate (ORR), disease control rate (DCR), and correlation of PD-L1 expression level and efficacy (ORR, PFS, and OS) (41). At the time of writing all patients have been recruited and the estimated Study completion date is April 15, 2022.

Given the recent demonstration that bevacizumab has efficacy in the front-line setting (8), a second multicenter randomized phase III trial was initiated comparing atezolizumab plus bevacizumab and standard chemotherapy versus bevacizumab and standard chemotherapy as firstline treatment for advanced MPM (BEAT-Meso). The aim of the BEAT-meso trial is to address whether the addition of the anti-PD-L1 antibody atezolizumab given in combination with standard chemotherapy plus bevacizumab improves the outcome in advanced treatment-naïve MPM patients. The trial is currently recruiting $(\mathrm{n}=320)$ patients who will be randomized (1:1) to receive either Bevacizumab $15 \mathrm{mg} / \mathrm{kg}$ intravenously on day 1 of every 3 -week cycle, plus 4-6 cycles of chemotherapy, or Atezolizumab 1,200 mg fixed dose intravenously on day 1 of every 3 -week cycle, plus Bevacizumab $15 \mathrm{mg} / \mathrm{kg}$, intravenously on day 1 of every 3-week cycle, plus 4-6 cycles of chemotherapy. Treatment will continue until either disease progression, or treatment is stopped at the request of the participant or treating doctor, or the participant withdraws consent. The primary outcome measures are PFS \& OS, and secondary outcomes include response rate, DCR, time to treatment failure, duration of response, safety and tolerability, patient reported outcome and quality of life (QoL). The estimated study completion date for this trial is December 31, 2024.

The results of these Phase III clinical trials are eagerly awaited.

\section{Checkpoint inhibitor Immunotherapy within the salvage setting}

A large body of trials have investigated (or are currently investigating) the potential use of checkpoint inhibitors within the second or third-line (salvage therapy) setting. The main trials are summarized in Table 3 and are discussed below in more detail.

\section{Single agent}

The first clinical trials of a checkpoint inhibitor in mesothelioma involved anti-CTLA4 therapy. Promising initial Phase I trials $(54,55)$ led to the phase II DETERMINE trial. This was a double-blind, placebo-controlled, phase $2 \mathrm{~b}$ trial conducted at 105 study centers across 19 countries in patients with unresectable pleural or peritoneal malignant mesothelioma who had progressed after one or two previous systemic treatments for advanced disease. Patients $(\mathrm{n}=571)$ were randomized (2:1) to receive intravenous tremelimumab $(10 \mathrm{mg} / \mathrm{kg})$ or placebo every 4 weeks for 7 doses and every 12 weeks thereafter until a treatment protocol discontinuation criterion was met. The primary endpoint was overall survival in the intention-to-treat population, but unfortunately this study was a negative study, as median overall survival in the intention-to-treat population did not differ between the treatment groups: 7.7 months (tremelimumab) $v s$. and 7.3 months (placebo) (46). 
Table 3 Salvage therapy checkpoint inhibitor trials in MPM discussed in this article

\begin{tabular}{|c|c|c|c|c|c|c|}
\hline Agent & Phase & Identifier & Target & Number of patients & Status & Reference \\
\hline Nivolumab & II & JapicCTI-163247 & Anti-PD-1 & $\mathrm{N}=34$ & Complete & $(45)$ \\
\hline Pembrolizumab & IB & NCT02054806 & Anti-PD-1 & $\mathrm{n}=25$ & Active, not recruiting & $(47)$ \\
\hline Pembrolizumab & II & NCT02399371 & Anti-PD-1 & $\mathrm{n}=65$ & Active, not recruiting & $(48)$ \\
\hline Nivolumab & II & NCT02497508 & Anti-PD-1 & $n=33$ & Completed & (49) \\
\hline Tremelimumab; durvalumab & ॥ & NCT02588131 & $\begin{array}{l}\text { Anti-CTLA4; } \\
\text { Anti-PD-L1 }\end{array}$ & $n=40$ & Unknown & (39) \\
\hline Avelumab & IB & NCT01772004 & Anti-PD-L1 & $\mathrm{n}=53$ & Active, not recruiting & $(51)$ \\
\hline Nivolumab & III & NCT03063450 & Anti-PD-1 & $n=336$ & Recruiting & (52) \\
\hline Pembrolizumab & III & NCT02991482 & Anti-PD-1 & $n=144$ & Active, not recruiting & (53) \\
\hline
\end{tabular}

MPM, malignant pleural mesothelioma.

Results from the Keynote-028 study involving pembrolizumab (anti-PD-1) treatments in mesothelioma were reported in 2017 (47). In this phase IB study, patients $(\mathrm{n}=25)$ with PD-L1-positive MPM must have either failed standard therapy or were unable to receive standard therapy, and received pembrolizumab $(10 \mathrm{mg} / \mathrm{kg}$ every 2 weeks) for up to 2 years or until confirmed progression or unacceptable toxicity. The median OS was 18 months. A recent analysis of the entire trial cohort $(n=475)$ found that T-cell-inflamed GEP, PD-L1 expression and/or tumor mutational burden was associated with a higher likelihood of response to therapy. Within this analysis of the $\mathrm{n}=25$ mesothelioma patients $n=19$ had GEP; $n=12$ PD-L1 positivity and $n=9$ had TMB data available (56). However, no subgroup analysis was available for the mesothelioma cohort alone.

The results of a phase II study of pembrolizumab conducted at the University of Chicago (Lead investigator Hedy Kindler). Having passed its interim objective a total of $\mathrm{n}=65$ patients were recruited. The results reported suggest that single-agent pembrolizumab in the salvage setting has robust activity in PD-L1 unselected mesothelioma, with no unexpected toxicities. Median OS was reported as 11.5 months, and increasing PD-L1 expression was associated with a trend towards a higher response rate and more durable PFS (48).

NivoMes was a Phase II trial of single agent nivolumab conducted on $(n=38)$ patients who had either disease recurrence or a failed chemotherapy regimen. Upon recruitment patients received Nivolumab $(3 \mathrm{mg} / \mathrm{kg}$ ) administered every 2 weeks intravenously for a maximum of 12 months or until disease progression or unacceptable toxicity. Overall response rates (ORR) of $24 \%$ were observed but no median OS was reported (49).

A Phase IB trial (JAVELIN) examined Avelumab in ( $\mathrm{n}=53)$ patients with unresectable mesothelioma that progressed after platinum and pemetrexed treatment (56). In the trial patients received avelumab, $10 \mathrm{mg} / \mathrm{kg}$, every 2 weeks until disease progression, unacceptable toxic effects, or withdrawal from the study. Overall the trial found that there was an acceptable safety profile coupled with a median OS of 10.7 months (51).

In 2018, the Japanese Ministry of Health, Labor and Welfare approved Nivolumab as a salvage therapy in mesothelioma based on the results of the MERIT trial. In this phase II trial patients received Nivolumab $240 \mathrm{mg}$ intravenously every 2 weeks until progressive disease or unacceptable toxicity (45). The primary endpoint was ORR by central assessment according to the Modified Response 
Evaluation Criteria in Solid Tumors, and was found to be 17.3 months (45).

One of the limitations of the smaller clinical trials conducted so far is that many of them are early-phase design, particularly the small number of patients enrolled, which limits data robustness and reduces the potential for subgroup analyses. Moreover several have single-arm nonrandomized design thus preventing any direct comparison with alternative treatment options in this disease setting. However, several Phase III trials are now running which should hopefully allow for better comparisons as detailed below.

A Phase III double-blind trial (CONFIRM) investigating Nivolumab in patients with relapsed mesothelioma is currently enrolling with an estimated completion date of July 2021 (52). Patients in this study must have received at least two prior lines of therapy (including patients who have had re-challenge with platinum/pemetrexed). Prior maintenance therapy is permitted but will not count as a line of treatment. In addition any prior lines of antineoplastic therapy, including chemotherapy, surgical resection of lesions, radiation therapy, must be completed at least 14 days prior to receiving study treatment. Patients will be randomized 2:1 (nivolumab:placebo) and stratified according to epithelioid/ non-epithelioid histology. They will receive either $240 \mathrm{mg}$ nivolumab as monotherapy or saline placebo as a $30-\mathrm{min}$ intravenous infusion for up to 12 months. The main of the CONFIRM trial will be to evaluate the efficacy, safety and cost-effectiveness of treatment with nivolumab in patients with relapsed mesothelioma. Its primary outcome measure will be OS (time to event), and secondary outcomes include OS according to PD-L1 status; PFS—-time to event; QoL; toxicity; treatment compliance and cost effectiveness (52).

A second Phase III trial (PROMISE-meso) is investigating whether treatment with pembrolizumab improves PFS compared to standard chemotherapy (gemcitabine or vinorelbine). In this trial of $(n=144)$ patients will be randomized (1:1) into either arm and will receive either pembrolizumab (at $200 \mathrm{mg}$ fixed dose intravenously every 3 weeks for a maximum or 2 years), or until disease progression or unacceptable toxicity, or until the patient declines further treatment. In the chemotherapy arm patients will receive either Gemcitabine (intravenously $1,000 \mathrm{mg} / \mathrm{m}^{2}$ ) or vinorelbine (intravenously $30 \mathrm{mg} / \mathrm{m}^{2}$, or by oral administration $60 / 80 \mathrm{mg} / \mathrm{m}^{2}$ ) chemotherapy on days 1 and 8 of every 3 -week cycle, and upon progression will be allowed to cross-over to receive pembrolizumab at progression, if cross-over criteria are met. The primary outcome measure in this trial will be PFS and in this regard, it has previously been noted that PFS has been shown to reflect OS in mesothelioma (57). At any rate secondary outcomes include OR, OS, toxicity and time to treatment failure. Interim results of this trial were disappointing and were recently reported (53). Median PFS was 2.5 months for the 73 patients who were randomly assigned to receive $200 \mathrm{mg}$ pembrolizumab every 3 weeks, after progressing on previous platinum-based chemotherapy. This did not differ significantly from the median PFS of 3.4 months observed among the 71 patients given single-agent chemotherapy, either gemcitabine or vinorelbine. Moreover, allowing for crossover yielded similar OS results, and as such it was concluded that pembrolizumab does not confer a progression free survival (PFS) or overall survival (OS) advantage in previously treated patients with advanced MPM (53). Whilst there did appear to be an improved ORR in unselected patients treated with pembrolizumab, it did not improve PFS or OS over single agent chemotherapy. As such it will be important to take on board the results of this trial moving forwards in the design and use of checkpoint inhibitors within the salvage setting.

\section{Combination therapy}

Recently, the results of the MAPS-2 trial have been reported (44). This prospective multi-center Phase II trial had randomized N=125 ECOG PS 0-1 MPM patients who had progressed upon receiving 1-2 prior treatment regimens which had to have included platinum-containing therapy. A non-comparative study it contained two arms, and patients were randomly allocated $(1: 1)$ to receive intravenous Nivolumab ( $3 \mathrm{mg} / \mathrm{kg}$ bodyweight) every 2 weeks, or intravenous Nivolumab (3 mg/kg every 2 weeks) plus intravenous Ipilimumab ( $1 \mathrm{mg} / \mathrm{kg}$ every 6 weeks), given until progression or unacceptable toxicity. ORs were 26$28 \%$ in the Nivolumab + Ipilimumab arm and $19 \%$ in the Nivolumab alone arm $(17,42,44)$. Median OS data observed was 11.9 months for the single therapy and 15.9 months for the combination (44).

The NIBIT-Meso-1 trial was an open-label, nonrandomized, phase II study (39). Patients enrolled in this study either had have refused a first line platinum-based chemotherapy, or had disease progression following one line of platinum-based therapy for advanced disease. Forty patients were enrolled and received at least one dose each of tremelimumab and durvalumab, and the combination of tremelimumab and durvalumab had a good safety 
Table 4 Immune-related markers expression; roles in anti-tumor immunity and candidate therapeutic agents in clinical development. Adapted from (59)

\begin{tabular}{|c|c|c|c|c|}
\hline Target/marker & Expression on Immune Cells & $\begin{array}{l}\text { Expression on } \\
\text { mesothelioma cells }\end{array}$ & $\begin{array}{l}\text { Function with respect to } \\
\text { anti-tumor immunity }\end{array}$ & Compound in development \\
\hline VISTA & $\begin{array}{l}\text { CD8+, CD4+ T-cells, Tregs, } \\
\text { NK cells, DC, monocytes, } \\
\text { macrophages, granulocytes }\end{array}$ & Yes & Co-inhibitory & CA-170 \\
\hline $\mathrm{B} 7 \mathrm{H} 3$ & $\begin{array}{l}\text { T-cells, antigen-presenting cells } \\
\text { (APC), NK-cells }\end{array}$ & Yes & $\begin{array}{l}\text { Co-stimulatory; co- } \\
\text { inhibitory }\end{array}$ & $\begin{array}{l}\text { Enoblituzumab; Orlotamab; } \\
\text { MGC018 }\end{array}$ \\
\hline TIM-3 & $\begin{array}{l}\text { CD8+, CD4+ T helper } 1 \text { cells } \\
\text { (Th1 cells), Tregs, NK cells, DC, } \\
\text { monocytes, macrophages }\end{array}$ & No & Co-inhibitory & $\begin{array}{l}\text { MBG453; TSR-022; LY3321367; } \\
\text { INCAGN02390; Sym023; } \\
\text { BGB-A425; LY3415244 }\end{array}$ \\
\hline
\end{tabular}

1, anti-LAG3 + anti-PD-1; ${ }^{2}$, anti-LAG3 + anti-CTLA4; ${ }^{3}$, anti-TIM3 + anti-PD-L1. MPM, malignant pleural mesothelioma; DC, dendritic cell.

profile with any treatment-related toxicity being generally manageable and reversible. Some activity was observed, and median OS was 16.6 months (39).

The INITIATE trial (50) was another open, prospective, single-arm phase II study which examined the combination of Ipilimumab and Nivolumab in MPM. For this trial of $\mathrm{n}=35$, patients had to have progressed after at least one line of platinum-containing chemotherapy. Patients received intravenous Nivolumab (240 mg every 2 weeks) plus intravenous Ipilimumab $(1 \mathrm{mg} / \mathrm{kg}$ every 6 weeks up to four times), and treatment was continued for up to 2 years or until confirmed progression or unacceptable toxicity. The safety profile observed was consistent with known data for this combination regimen, and was well tolerated. At the time of reporting, median OS had not yet been reached but was estimated, with $95 \%$ confidence, to exceed 12.7 months (58).

Other studies however with combined checkpoint inhibitors have had disappointing results. For example, a Phase 2 Study of Durvalumab in combination with Tremelimumab in MPM has been carried out at the Dana Farber Cancer Institute (NCT03075527). Designed to explore the activity of combined CTLA-4 + PD-L1 immune checkpoint inhibition using Tremelimumab plus Durvalumab in previously-treated MPM, at interim analysis (43), the study did not meet its primary endpoint, and at the present point in time is currently suspended.

The results of these trials continue to support the further development of checkpoint inhibitors as both single agents or as combination therapies in mesothelioma.

\section{Checkpoint inhibitors are not just PD-1/PD-L1 and CTLA4 what else is coming down the track for MPM}

However, checkpoint inhibitors and anti-tumor immunity are not restricted to just the three markers currently described. Many other potential immunotherapy targets have been identified and significant industrial efforts have led to the development of numerous candidate molecules recently reviewed in detail by Chrétien et al. (59) (Table 4). In the following sections we describe some of those with potential utility for use in mesothelioma.

\section{VISTA}

VISTA (also known as VSIR or B7H5) is an immune- 


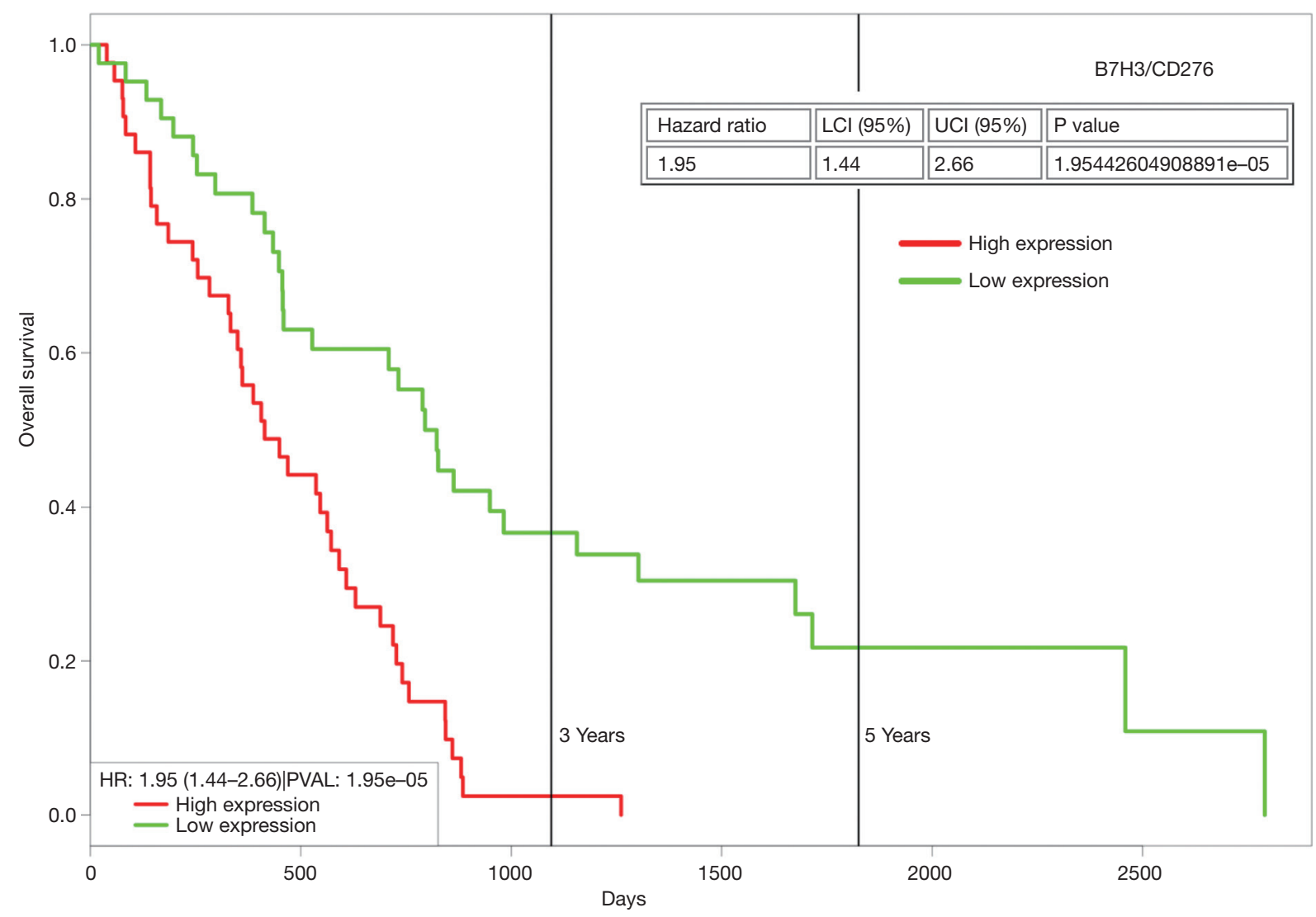

Figure 1 High expression of B7H3 (CD276) expression/is associated with poorer overall survival in mesothelioma. In silico analysis of OS for B7H3 in the TCGA mesothelioma dataset with the cohort divided at the median of gene expression. Analysis was conducted using ProgGeneV2 (65), and high expression of B7H3 (CD276) was associated with a significantly worse $\left(\mathrm{P}=1.9 \times 10^{-5}\right)$ overall survival.

checkpoint gene which was first reported as having strong expression in epithelioid MPM, above and beyond that seen in other solid cancers, with obvious implications for the immune response to MPM and for its immunotherapy (60). A subsequent study has confirmed that VISTA expression is higher in epithelioid subtype (61). A small molecule inhibitor against VISTA (CA-170) (62) is currently in a Phase I clinical trial (NCT02812875) in adult patients with advanced solid tumors or lymphomas who have progressed or are non-responsive to available therapies and for which no standard therapy exists, and includes mesothelioma as an eligible tumor. This trial has finished recruiting and study completion is expected in January 2020.

\section{B7H3}

B7H3 (also known as CD276) is another candidate checkpoint, whose expression has been observed in mesothelioma (63). The pharmaceutical company Macrogenics has several B7H3 compounds (Enoblituzumab/ MGA271; Orlotamab/MGD009; MGC018) in active clinical trials. In 2018, Macrogenics reported that expression of B7H3 was positive in $41 / 44$ mesothelioma samples tested, and of these 39/44 expressed B7H3 highly as tested by IHC (64). The histological subtype of the mesothelioma specimens examined was not provided. Using ProgeneV2 (65) to analyze the TCGA mesothelioma dataset for $\mathrm{B} 7 \mathrm{H} 3$, it is possible to stratify patients for OS based on median expression. Those patients with high expression of $\mathrm{B} 7 \mathrm{H} 3$ mRNA show worse OS (Figure 1), and as such it may be possible to utilize $\mathrm{B} 7 \mathrm{H} 3$ as a potential biomarker for patient stratification for checkpoint inhibitor therapy targeting B7H3.

\section{$L A G-3$}

Lymphocyte activation gene-3 (LAG-3) is a T-cell 
inhibitory receptor that suppresses both T-cell activation and cytokine secretion, thereby ensuring immune homeostasis (66). Immunotherapies and agents targeting LAG-3 are in active clinical trials (59), and combination immunotherapy of anti-LAG-3 and anti-PD-1 has shown exciting efficacy in fighting PD-1 resistance (66). There is no expression of LAG-3 on mesothelial cells themselves (67), but high levels of LAG-3 can be detected in pleural effusions of patients with mesothelioma $(68,69)$, and tumor infiltrating lymphocytes (TILs) in pleural effusions from patients with mesothelioma express high levels of this receptor (70).

\section{TIM-3}

T-Cell Immunoglobulin and Mucin Domains containing protein 3 (TIM-3) is widely expressed in a variety of immune cells where it affects both innate and adaptive immune response by regulating immune cell function, thus affecting tumor occurrence and development (71). In mesothelioma, double-positive PD-1+/TIM-3+ CD8+ T cells are more commonly found on PD-L1-positive tumors (72). Moreover, an analysis of a small cohort of patients treated with anti-CTLA4 demonstrated that improved OS was correlated with low TIM-3+ CD8+ T-cell frequency (73), raising the possibility that we may be able to refine our ability to predict patients that will respond to specific immune checkpoint blockade strategies including those that target TIM-3 (72).

\section{OX40/OX40L}

These are members of the TNF receptor superfamily (TNFRSF), and are key co-stimulators of $\mathrm{T}$ cells during infection, and there has been an increasing interest in harnessing these receptors to augment tumor immunity. OX40 (TNFRSF4) and OX40L (TNFSF4) have been implicated in mesothelioma. In a recent study of an animal model of mesothelioma, tumor resident regulatory T-cells were shown to co-express high levels of CTLA4 and OX40 on a large proportion of cells. Individually targeting OX40 generated an effective response against tumor development, and was found to be synergistic with anti-CTLA4 agents (74). Whilst there appears to be little information as regards OX40L in mesothelioma, analysis of the TCGA dataset demonstrates that high expression is associated with poorer OS (Figure 2).

\section{Other immune-related markers}

Other immune-related markers which have been shown to have potential utility in mesothelioma include TLR9 (75), and GITR/TNFRSF18 (74).

In summary the wealth of candidate agents currently under investigation coupled with the existing published data from patients with mesothelioma suggest that combination immune checkpoint inhibitor therapy, whilst still in its infancy may work synergistically to induce strong, durable immunity responses in mesothelioma, and further investigations are warranted.

\section{Oncolytic virus therapy}

One area which is currently emerging as having significant potential in mesothelioma is that of oncolytic viral (OV) therapy. Such a strategy typically involves the use of viruses that combine tumor-specific cell lysis together with immune stimulation, therefore acting as potential in situ tumor vaccines (76). Moreover, OVs are being considered as a potential therapeutic option for patients who do not respond or fail to achieve durable responses following treatment with immune checkpoint inhibitors (77), and several pre-clinical studies have now emerged showing that OVs have potential to target mesothelioma (78-87).

One of the first OV developed which has been used in clinical trials of mesothelioma involves the herpes simplex virus (HSV)-1716 (88). In 1998, the results of a Phase I clinical trial of an adenovirus based HSV1716 OV were reported (89). This trial of $n=21$ patients demonstrated that intra-pleural administration of an adenoviral vector containing the HSVtk gene is well tolerated and resulted in efficient gene transfer when delivered at high doses. No patient responses were described. Long-term follow up for $\mathrm{n}=11$ of the patients treated with this OV were presented in 2005, but there were no long-term survivors ( $>5$ years) among any of the patients who received the OV. All 13 patients died from the natural progression of their underlying mesothelioma (90). A different (HSV)-1716 OV utilized by the authors in a separate trial did however show promising long-term survival in a cohort of patients (90).

A separate OV SEPREHVIR ${ }^{\circledR}$ (HSV1716) developed by Virttu/Sorrento is in Phase I/IIA clinical trial in mesothelioma, and the interim results of this trial were presented in 2017 (91). In this trial $n=12$ patients received OV. Of these, 6 patients had stable disease, and median 

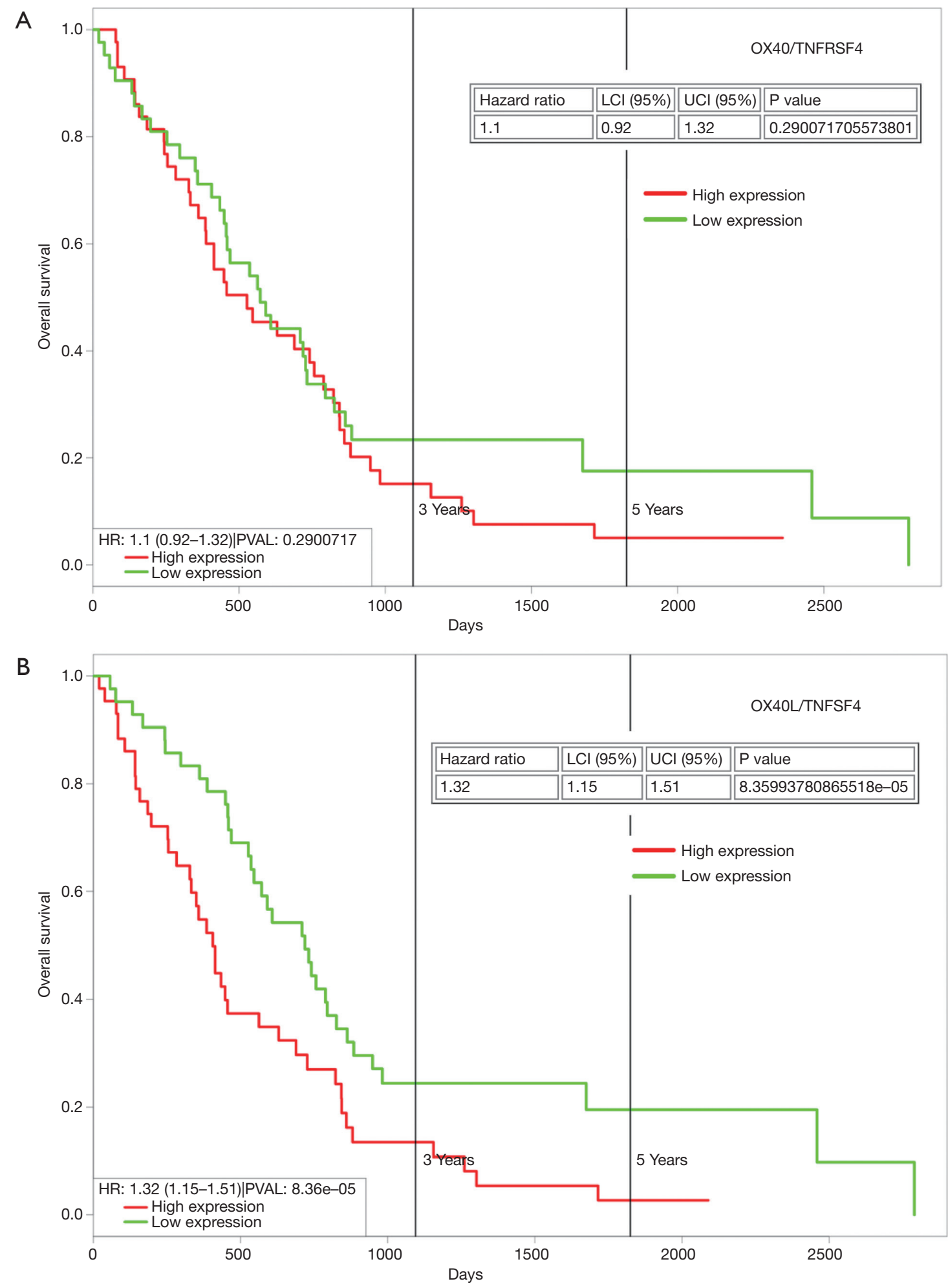

Figure 2 High expression of OX40L but not OX40 is associated with poorer overall survival in mesothelioma. In silico analysis of OS was carried out using ProgGeneV2 (65) on the TCGA mesothelioma dataset with the cohort divided at the median of gene expression. (A) No significant OS difference was observed for OX40; (B) when stratified high expression of OX40L was associated with a significantly worse $\left(\mathrm{P}=8.4 \times 10^{-5}\right)$ overall survival. 
survival was 15 months for all patients (91).

Returning to GM-CSF, a dual targeting, chimeric oncolytic adenovirus, coding for human GM-CSF (ONCOS-102) has been developed, and pre-clinical evaluation in an experimental mesothelioma model showed potential clinical efficacy, which was found to be synergistic when combined with chemotherapy (92). Subsequently a phase IB/II clinical trial (clinicaltrials.gov NCT02879669) of ONCOS-102 in mesothelioma was initiated and has recently completed patient accrual. The trial itself is assessing the combination of ONCOS-102 and SoC chemotherapy (pemetrexed and cisplatin) vs. SoC chemotherapy alone in first or second/third line patients with unresectable MPM. Interim reports on the trial were reported in 2018 (93), with no safety issues reported, strong innate and adaptive immune activation and 50\% DCR for the six patients in the phase IB safety lead-in cohort. Enrolment of 25 patients into the randomized phase II part of the trial has now been completed (94), and final results are expected towards the end of 2019.

Intriguingly, in the evaluation of the ONCOS-102 phase I safety study, it was noted that this OV could potentially induce PD-L1. Moreover, a clear post-treatment induction of PD-L1 expression was observed in the tumors of two mesothelioma patients, with PD-L1 histoscore increasing from baseline levels of 17 and 1 to 47 and 23 after treatment, respectively (95). Whilst a clinical study based on this observation has not been initiated in MPM, Targovax has begun a phase I/II trial investigating the safety, biologic and anti-tumor activity of ONCOS-102 in combination with Imfinzi (durvalumab, anti-PD-L1) in patients with advanced peritoneal malignancies who have failed prior standard chemotherapy and have histologically confirmed platinum-resistant or refractory epithelial ovarian or colorectal cancer (ClinicalTrials.Gov NCT02963831). The results of these initial studies will hopefully confirm the potential of ONCOS-102 as an immune-sensitizing agent for combinatory therapies with checkpoint inhibitors.

Other OV strategies currently being assessed include dl922-947 a second-generation adenoviral mutant bearing a 24-bp deletion in E1A-Conserved Region 2. This region binds to and inactivates $\mathrm{pRb}$, dissociating the pRb-E2F complex and driving $\mathrm{S}$ phase entry, and has shown preclinical efficacy in MPM (78). Another study involving an Oncolytic Measles Virotherapy is currently in a Phase I clinical trial (clinical trials.gov NCT NCT0150317), and has an estimated completion date in December 2019.

One issue which can affect oncolytic virus mediated therapy is the development of virus-neutralizing antibodies, but selective re-targeting of these is emerging as a novel measure to counteract this, and can be successfully combined with checkpoint inhibitors (96).

\section{Chimeric antigen receptors (CAR)-T therapies and dendritic cell immunotherapy}

CARs couple the HLA-independent binding of a cell surface target to the delivery of a tailored T-cell activating signal (97), by recognizing and binding to a specific tumorassociated antigen and have emerged as exciting new therapeutic opportunity particularly in leukemias (98).

The potential to use CAR-T therapy in mesothelioma has been explored fairly extensively, and pre-clinical models using mesothelin (MSLN) (99,100), FAP (101), cMET (97), pan-ErbB (102) and other targets have been extensively tested $(98,103)$.

The initial clinical trial (Clinicaltrials.gov NCT01355965) of an intravenous delivery of MSLN-targeted CARs was evaluated in a Phase I clinical trial of solid tumors (104), and included three patients with MPM. All three patients of these patients developed an antibody response to the murine component of this CAR, with one patient developing an anaphylactic reaction. Based on these results a second generation CD28-costimulated MSLN CAR was developed which incorporated an Icaspase-9 safety gene (IcasM28z). A Phase I clinical trial of this was initiated (clinicaltrials.gov NCT02414269) and included n=19 MPM patients. In this trial patients also received anti-PD1 checkpoint blockade agents (1-21 cycles), off protocol. Responses were gauged using metabolic response on PET scan. The best response among the 19 MPM patients (13 patients received antiPD1 agent; PD-L1 $<10 \%$ in all except 1 ) was -2 patients had complete (60 and 32 weeks ongoing); 5 demonstrated partial response and 4 had stable disease using PET, but CT-scans were not performed (105). Various other CAR-T trials utilizing MSLN have been trialed, but few results for these have been reported. One trial using a lentiviral transduction based approach (clinicaltrials. gov NCT02159716) was completed. However, in this trial which included mesothelioma patients, no clinical responses were observed (98).

A clinical trial of a single fixed dose of adoptively transferred FAP-specific CD8 positive re-directed T cells given in the pleural effusion in mesothelioma (clinicaltrials. gov NCT01722149) has completed recruitment, and the estimated completion of the study will be in July, 2019. 
One factor which may currently limit the use of CAR-T strategies in solid tumors could be the issue of T-Cell exhaustion, a state of $\mathrm{T}$ cell dysfunction that arises during chronic exposure to inhibitory signals encountered in the tumor microenvironment or activation-induced upregulation of co-inhibitory pathways that reduces their cytolytic and proliferative functions (106). However, a recent study has suggested that checkpoint inhibitors may therefore be an effective strategy for improving the potency of CAR T cell therapies in this regard (107).

Dendritic cell therapy is a cell-based vaccination approach used to initiate an anti-tumor immune response. Initial DC based approaches in mesothelioma using autologous tumor lysate loaded DCs showed promising long-lasting clinical responses with survival up to 66 months post treatment (108-112). The disadvantage of autologous DC approaches is that it is time-consuming and may not often generate sufficient amounts of the required quality. To circumvent this an allogenic tumor lysate-based approach was developed (113) and a Phase I clinical trial MesoCancerVa (NCT02395679) recently completed. In this trial, no dose-limiting toxicities were established and radiographic responses were observed. The median PFS was 8.8 months and median OS was not reached at a median follow-up of 22.8 months (113). These promising results have led to the establishment of the Phase II/III DENIM trial (NCT03610360) which aims to recruit $\mathrm{n}=230$ patients to examine the OS in patients treated with DCs loaded with this allogeneic tumor cell lysate as maintenance treatment after chemotherapy (114).

\section{Outstanding questions yet to be addressed fully}

Despite the significant strides made in cancer immunotherapy, there still remain other outstanding questions.

\section{Patient etbnicity}

As has been learned from EGFR TKI therapy in non-small cell lung cancer (NSCLC), ethnicity can play an important role in patient response (115). The majority of initial clinical trials for checkpoint inhibitors were carried out primarily on Caucasians, but usually included a small portion of Asian participants, and results were calculated and interpreted for the entire included subjects without any race-specific conclusions. This issue has been raised and commented on recently by Shi and colleagues particularly with regard to the management of adverse events (116). Nevertheless, the results of the MERIT trial suggests that this is an area which should not be ignored, and issues of ethnicity may yet play a role in the future of immunotherapy in mesothelioma.

\section{Is there an issue of Hyper-progression in mesothelioma?}

It is now established that hyper-progression can occur for a subset of patients undergoing checkpoint inhibitor therapy, which can emerge either when the patient is undergoing therapy (117), or which can emerge post-therapy (118). The estimated occurrence of this varies but runs to about $10 \%$ to $20 \%$ of treated cases (119). Whilst there is little evidence that hyper-progression occurs during treatments of mesothelioma, in the DREAM trial, two patients were reported as showing pseudo-progression, within the first $10-15$ weeks of therapy followed by responses (40).

In a recent editorial on this topic key issues remain such as: why it occurs; is it simply a lead-time bias phenomenon; does it have a strong biological basis such as clonal selection; can we identify and predict those in whom it will occur; and if it can be stopped by additional therapies (119). As more and more clinical trials of immunotherapies complete in mesothelioma, vigilance will be required to assess if hyperprogression does occur.

\section{How to make a cold tumor bot?}

A significant challenge facing all immunotherapy based approaches is the issue of Tumor T-cell infiltration. Those tumors which have a poor infiltration are often characterized as so-called "cold tumors" and more resistant to immunotherapy (120). How therefore can we improve on mesothelioma immunotherapies to increase the proportion of responsive patients? Aside from targeting additional immunomodulatory molecules, it may be possible to use cytokines such as IL-2 and growth factors such as GMCSF, or oncolytic viruses etc., as discussed in previous sections along with other therapies such as radiotherapy, photodynamic therapy electro-chemotherapy etc. (13).

\section{Epigenetics to prime immunotherapy?}

One such avenue may be to target the epigenetic machinery to elicit such responses sometimes described as immunoprobing (121). In this setting clinically relevant non-toxic doses of epigenetic targeting agents are used to induce antitumor "memory" responses including 
immunomodulatory pathways (122). It is well established that PD-1/PD-L1 can be affected in cells following treatment with histone deacetylase inhibitors (HDACi) (123). Several lines of evidence in NSCLC suggest that addition of an HDACi can increase tumor antigen presentation, decrease immune suppressive cell types and augment checkpoint inhibitor therapy (124). Furthermore, a recent phase 1/1b study of pembrolizumab plus vorinostat (a HDACi) in advanced/metastatic NSCLC found that this combination was well tolerated and demonstrated preliminary antitumor activity despite progression on prior checkpoint inhibitor treatment (125), while encouraging responses to such a strategy were also observed in the ENCORE-601 trial which combined Entinostat (a HDACi) with Pembrolizumab (NCT02437136) (126,127).

These activities are not just limited to histone deacetylases as similar effects have been observed for DNA methyltransferase inhibitors $(128,129)$, and bromodomain inhibitors (130).

Whilst the initial Phase III trial of Vorinostat In mesothelioma failed (131), it would be very interesting to re-examine the data from this trial to determine if any increases in PD-L1 expression were observed. More recently, new HDACi in pre-clinical development have shown that they can induce PD-L1 in mesothelioma cell line in vitro (132), suggesting that including HDACi could potentially be a combinatorial approach to immunotherapy in mesothelioma.

\section{Other chemotherapies to prime immunotherapy?}

Other strategies could also potentially be used in this regard, including standard chemotherapies. Indeed gemcitabine was recently shown to synergize with checkpoint inhibitors and overcome resistance in a preclinical mouse model of mesothelioma and in two patients treated with this combination (133). However, it may well be that this observation could potentially be related to the known effects of gemcitabine on DNA methylation in mesothelioma cell lines resulting in the induction of PD-L1 (134).

\section{Targeting tumour-infiltrating cells (Tregs)}

Analogous to other neoplasms, a more precise knowledge of Tregs could identify a specific predictive signature which may provide novel actionable targets for immunotherapy (135). Preliminary findings by us provide clear evidence that Tregs in MPM show a particularly clear-cut immuno-suppressive signature and this should be certainly considered in the design of new therapeutic approaches to treat MPM (Mutti et al., manuscript in preparation).

\section{Summary}

The previous sections have discussed in depth the current clinical trials involving immunotherapy in MPM. Despite intensive research, breakthroughs in the treatment of this cancer have been limited, and the lack of interest and funding has certainly delayed any dramatic progress to date. Nevertheless, the advent of more recent cancer immunotherapy options has shed new light onto potential treatments for the future. In this regard we await the results of critical checkpoint inhibitor phase II/III trials with great interest in the hope that the potential significant advances in outcome will be achieved.

\section{Acknowledgments}

The authors would like to acknowledge those authors whom we were unable to cite in this article.

Funding: None.

\section{Footnote}

Conflicts of Interest: The authors have no conflicts of interest to declare.

Ethical Statement: The authors are accountable for all aspects of the work in ensuring that questions related to the accuracy or integrity of any part of the work are appropriately investigated and resolved.

Open Access Statement: This is an Open Access article distributed in accordance with the Creative Commons Attribution-NonCommercial-NoDerivs 4.0 International License (CC BY-NC-ND 4.0), which permits the noncommercial replication and distribution of the article with the strict proviso that no changes or edits are made and the original work is properly cited (including links to both the formal publication through the relevant DOI and the license). See: https://creativecommons.org/licenses/by-nc$\mathrm{nd} / 4.0 \%$.

\section{References}

1. Carbone M, Adusumilli PS, Alexander HR Jr, et al. 
Mesothelioma: Scientific clues for prevention, diagnosis, and therapy. CA Cancer J Clin 2019;69:402-29.

2. Carbone M, Baris YI, Bertino P, et al. Erionite exposure in North Dakota and Turkish villages with mesothelioma. Proc Natl Acad Sci U S A 2011;108:13618-23.

3. Carbone M, Ly BH, Dodson RF, et al. Malignant mesothelioma: facts, myths, and hypotheses. J Cell Physiol 2012;227:44-58.

4. Maher B. Epidemiology: Fear in the dust. Nature 2010;468:884-5.

5. Kindler HL, Ismaila N, Armato SG, 3rd, et al. Treatment of Malignant Pleural Mesothelioma: American Society of Clinical Oncology Clinical Practice Guideline. J Clin Oncol 2018;36:1343-73.

6. Vogelzang NJ, Rusthoven JJ, Symanowski J, et al. Phase III study of pemetrexed in combination with cisplatin versus cisplatin alone in patients with malignant pleural mesothelioma. J Clin Oncol 2003;21:2636-44.

7. Strizzi L, Catalano A, Vianale G, et al. Vascular endothelial growth factor is an autocrine growth factor in human malignant mesothelioma. J Pathol 2001;193:468-75.

8. Zalcman G, Mazieres J, Margery J, et al. Bevacizumab for newly diagnosed pleural mesothelioma in the Mesothelioma Avastin Cisplatin Pemetrexed Study (MAPS): a randomised, controlled, open-label, phase 3 trial. Lancet 2016;387:1405-14.

9. FDA Approves the NovoTTF-100LTM System in Combination with Chemotherapy for the Treatment of Malignant Pleural Mesotheliom. Business Wire 2019.

10. Mun EJ, Babiker HM, Weinberg U, et al. Tumor-Treating Fields: A Fourth Modality in Cancer Treatment. Clin Cancer Res 2018;24:266-75.

11. Fabian D, Guillermo Prieto Eibl MDP, Alnahhas I, et al. Treatment of Glioblastoma (GBM) with the Addition of Tumor-Treating Fields (TTF): A Review. Cancers (Basel) 2019. doi: 10.3390/cancers11020174.

12. Ceresoli GL, Aerts JG, Dziadziuszko R, et al. Tumour Treating Fields in combination with pemetrexed and cisplatin or carboplatin as first-line treatment for unresectable malignant pleural mesothelioma (STELLAR): a multicentre, single-arm phase 2 trial. Lancet Oncol 2019;20:1702-9.

13. Locy H, de Mey S, de Mey W, et al. Immunomodulation of the Tumor Microenvironment: Turn Foe Into Friend. Front Immunol 2018;9:2909.

14. Bakker E, Guazzelli A, Ashtiani F, et al. Immunotherapy advances for mesothelioma treatment. Expert Rev Anticancer Ther 2017;17:799-814.
15. Decker WK, da Silva RF, Sanabria MH, et al. Cancer Immunotherapy: Historical Perspective of a Clinical Revolution and Emerging Preclinical Animal Models. Front Immunol 2017;8:829.

16. Ledford H, Else H, Warren M. Cancer immunologists scoop medicine Nobel prize. Nature 2018;562:20-1.

17. Nowak AK, McDonnell A, Cook A. Immune checkpoint inhibition for the treatment of mesothelioma. Expert Opin Biol Ther 2019;19:697-706.

18. Parra HS, Tixi L, Latteri F, et al. Combined regimen of cisplatin, doxorubicin, and alpha-2b interferon in the treatment of advanced malignant pleural mesothelioma: a Phase II multicenter trial of the Italian Group on Rare Tumors (GITR) and the Italian Lung Cancer Task Force (FONICAP). Cancer 2001;92:650-6.

19. Soulié P, Ruffié P, Trandafir L, et al. Combined systemic chemoimmunotherapy in advanced diffuse malignant mesothelioma. Report of a phase I-II study of weekly cisplatin/interferon alfa-2a. J Clin Oncol 1996;14:878-85.

20. Upham JW, Musk AW, van Hazel G, et al. Interferon alpha and doxorubicin in malignant mesothelioma: a phase II study. Aust N Z J Med 1993;23:683-7.

21. Monnet I, Breau JL, Moro D, et al. Intrapleural infusion of activated macrophages and gamma-interferon in malignant pleural mesothelioma: a phase II study. Chest 2002;121:1921-7.

22. Sterman DH, Alley E, Stevenson JP, et al. Pilot and Feasibility Trial Evaluating Immuno-Gene Therapy of Malignant Mesothelioma Using Intrapleural Delivery of Adenovirus-IFNalpha Combined with Chemotherapy. Clin Cancer Res 2016;22:3791-800.

23. Sterman DH, Haas A, Moon E, et al. A trial of intrapleural adenoviral-mediated Interferon-alpha2b gene transfer for malignant pleural mesothelioma. Am J Respir Crit Care Med 2011;184:1395-9.

24. Sterman DH, Recio A, Haas AR, et al. A phase I trial of repeated intrapleural adenoviral-mediated interferon-beta gene transfer for mesothelioma and metastatic pleural effusions. Mol Ther 2010;18:852-60.

25. Dirix LY, van Meerbeeck J, Schrijvers D, et al. A phase II trial of dose-escalated doxorubicin and ifosfamide/mesna in patients with malignant mesothelioma. Ann Oncol 1994;5:653-5.

26. Davidson JA, Musk AW, Wood BR, et al. Intralesional cytokine therapy in cancer: a pilot study of GM-CSF infusion in mesothelioma. J Immunother 1998;21:389-98.

27. Kosty MP, Herndon JE 2nd, Vogelzang NJ, et al. Highdose doxorubicin, dexrazoxane, and GM-CSF in malignant 
mesothelioma: a phase II study-Cancer and Leukemia Group B 9631. Lung Cancer 2001;34:289-95.

28. Powell A, Creaney J, Broomfield S, et al. Recombinant GM-CSF plus autologous tumor cells as a vaccine for patients with mesothelioma. Lung Cancer 2006;52:189-97.

29. Khanna S, Graef S, Mussai F, et al. Tumor-Derived GMCSF Promotes Granulocyte Immunosuppression in Mesothelioma Patients. Clin Cancer Res 2018;24:2859-72.

30. Murthy V, Katzman D, Sterman DH. Intrapleural immunotherapy: An update on emerging treatment strategies for pleural malignancy. Clin Respir J 2019;13:272-9.

31. Tsao AS, Lindwasser OW, Adjei AA, et al. Current and Future Management of Malignant Mesothelioma: A Consensus Report from the National Cancer Institute Thoracic Malignancy Steering Committee, International Association for the Study of Lung Cancer, and Mesothelioma Applied Research Foundation. J Thorac Oncol 2018;13:1655-67.

32. Malignant Mesothelioma Treatment (Adult) (PDQ®): Health Professional Version. PDQ Cancer Information Summaries [Internet]. Bethesda (MD): National Cancer Institute (US); 2002.

33. Abdel-Rahman O, Elsayed Z, Mohamed H, et al. Radical multimodality therapy for malignant pleural mesothelioma. Cochrane Database Syst Rev 2018;1:CD012605.

34. Friedberg JS, Culligan MJ, Tsao AS, et al. A Proposed System Toward Standardizing Surgical-Based Treatments for Malignant Pleural Mesothelioma, From the Joint National Cancer Institute-International Association for the Study of Lung Cancer-Mesothelioma Applied Research Foundation Taskforce. J Thorac Oncol 2019;14:1343-53.

35. Gomez DR, Rimner A, Simone CB II, et al. The Use of Radiation Therapy for the Treatment of Malignant Pleural Mesothelioma: Expert Opinion from the National Cancer Institute Thoracic Malignancy Steering Committee, International Association for the Study of Lung Cancer, and Mesothelioma Applied Research Foundation. J Thorac Oncol 2019;14:1172-83.

36. Wei SC, Duffy CR, Allison JP. Fundamental Mechanisms of Immune Checkpoint Blockade Therapy. Cancer Discov 2018;8:1069-86.

37. O'Donnell JS, Hoefsmit EP, Smyth MJ, et al. The Promise of Neoadjuvant Immunotherapy and Surgery for Cancer Treatment. Clin Cancer Res 2019;25:5743-51.

38. Kindler H, Ferguson M, Tan YH, et al. P2.06-029 Pilot Window-Of-Opportunity Study of Pembrolizumab in Patients with Resectable Malignant Pleural Mesothelioma
(MPM): Topic: Mesothelioma and SCLC. J Thorac Oncol 2017;12:S1089.

39. Calabrò L, Morra A, Giannarelli D, et al. Tremelimumab combined with durvalumab in patients with mesothelioma (NIBIT-MESO-1): an open-label, non-randomised, phase 2 study. Lancet Respir Med 2018;6:451-60.

40. Nowak A, Kok P, Lesterhuis W, et al. OA08.02 DREAM - A Phase 2 Trial of Durvalumab with First Line Chemotherapy in Mesothelioma: Final Result. J Thorac Oncol 2018;13:S338-S9.

41. Zalcman G, Peters S, Mansfield AS, et al. Checkmate 743: A phase 3, randomized, open-label trial of nivolumab (nivo) plus ipilimumab (ipi) vs pemetrexed plus cisplatin or carboplatin as first-line therapy in unresectable pleural mesothelioma. J Clin Oncol 2017;35:abstr TPS8581.

42. Forde PM, Scherpereel A, Tsao AS. Use of Immune Checkpoint Inhibitors in Mesothelioma. Curr Treat Options Oncol 2019;20:18.

43. Venkatraman D, Anderson A, Digumarthy S, et al. Phase 2 study of tremelimumab plus durvalumab for previouslytreated malignant pleural mesothelioma (MPM). J Clin Oncol 2019;37:abstr 8549.

44. Scherpereel A, Mazieres J, Greillier L, et al. Nivolumab or nivolumab plus ipilimumab in patients with relapsed malignant pleural mesothelioma (IFCT-1501 MAPS2): a multicentre, open-label, randomised, non-comparative, phase 2 trial. Lancet Oncol 2019;20:239-53.

45. Okada M, Kijima T, Aoe K, et al. Clinical Efficacy and Safety of Nivolumab: Results of a Multicenter, Openlabel, Single-arm, Japanese Phase II study in Malignant Pleural Mesothelioma (MERIT). Clin Cancer Res 2019;25:5485-92.

46. Maio M, Scherpereel A, Calabro L, et al. Tremelimumab as second-line or third-line treatment in relapsed malignant mesothelioma (DETERMINE): a multicentre, international, randomised, double-blind, placebocontrolled phase 2b trial. Lancet Oncol 2017;18:1261-73.

47. Alley EW, Lopez J, Santoro A, et al. Clinical safety and activity of pembrolizumab in patients with malignant pleural mesothelioma (KEYNOTE-028): preliminary results from a non-randomised, open-label, phase $1 \mathrm{~b}$ trial. Lancet Oncol 2017;18:623-30.

48. Desai A, Karrison T, Rose B, et al. OA08.03 Phase II Trial of Pembrolizumab (NCT02399371) In Previously-Treated Malignant Mesothelioma (MM): Final Analysis. J Thorac Oncol 2018;13:S339.

49. Quispel-Janssen J, van der Noort V, de Vries JF, et al. Programmed Death 1 Blockade With Nivolumab in 
Patients With Recurrent Malignant Pleural Mesothelioma. J Thorac Oncol 2018;13:1569-76.

50. Disselhorst MJ, Quispel-Janssen J, Lalezari F, et al. Ipilimumab and nivolumab in the treatment of recurrent malignant pleural mesothelioma (INITIATE): results of a prospective, single-arm, phase 2 trial. Lancet Respir Med 2019;7:260-70.

51. Hassan R, Thomas A, Nemunaitis JJ, et al. Efficacy and Safety of Avelumab Treatment in Patients With Advanced Unresectable Mesothelioma: Phase 1b Results From the JAVELIN Solid Tumor Trial. JAMA Oncol 2019;5:351-7.

52. Fennell DA, Kirkpatrick E, Cozens K, et al. CONFIRM: a double-blind, placebo-controlled phase III clinical trial investigating the effect of nivolumab in patients with relapsed mesothelioma: study protocol for a randomised controlled trial. Trials 2018;19:233.

53. Popat S, Curioni-Fontecedro A, Polydoropoulou V, et al. LBA91_PRA multicentre randomized phase III trial comparing pembrolizumab $(\mathrm{P})$ vs single agent chemotherapy (CT) for advanced pre-treated malignant pleural mesothelioma (MPM): Results from the European Thoracic Oncology Platform (ETOP 9-15) PROMISEmeso trial. Ann Oncol 2019. doi: 10.1093/annonc/ mdz394.091.

54. Calabrò L, Morra A, Fonsatti E, et al. Tremelimumab for patients with chemotherapy-resistant advanced malignant mesothelioma: an open-label, single-arm, phase 2 trial. Lancet Oncol 2013;14:1104-11.

55. Calabrò L, Morra A, Fonsatti E, et al. Efficacy and safety of an intensified schedule of tremelimumab for chemotherapy-resistant malignant mesothelioma: an open-label, single-arm, phase 2 study. Lancet Respir Med 2015;3:301-9.

56. Ott PA, Bang YJ, Piha-Paul SA, et al. T-Cell-Inflamed Gene-Expression Profile, Programmed Death Ligand 1 Expression, and Tumor Mutational Burden Predict Efficacy in Patients Treated With Pembrolizumab Across 20 Cancers: KEYNOTE-028. J Clin Oncol 2019;37:318-27.

57. Wang X, Hodgson L, George SL, et al. Validation of Progression-Free Survival as a Surrogate Endpoint for Overall Survival in Malignant Mesothelioma: Analysis of Cancer and Leukemia Group B and North Central Cancer Treatment Group (Alliance) Trials. Oncologist 2017;22:189-98.

58. Bibby A, Maskell N. Checkpoint inhibitors in mesothelioma: hope for the future? Lancet Oncol 2019;20:172-4.

59. Chrétien S, Zerdes I, Bergh J, et al. Beyond PD-1/PD-
L1 Inhibition: What the Future Holds for Breast Cancer Immunotherapy. Cancers (Basel) 2019. doi: 10.3390/ cancers11050628.

60. Hmeljak J, Sanchez-Vega F, Hoadley KA, et al. Integrative Molecular Characterization of Malignant Pleural Mesothelioma. Cancer Discov 2018;8:1548-65.

61. Chung YS, Kim M, Cha YJ, et al. Expression of V-set immunoregulatory receptor in malignant mesothelioma. Mod Pathol 2020;33:263-70.

62. Musielak B, Kocik J, Skalniak L, et al. CA-170 - A Potent Small-Molecule PD-L1 Inhibitor or Not? Molecules 2019. doi: 10.3390/molecules24152804.

63. Calabrò L, Sigalotti L, Fonsatti E, et al. Expression and regulation of B7-H3 immunoregulatory receptor, in human mesothelial and mesothelioma cells: immunotherapeutic implications. J Cell Physiol 2011;226:2595-600.

64. Aggarwal C, Joshua A, Ferris R, et al. 33rd Annual Meeting \& Pre-Conference Programs of the Society for Immunotherapy of Cancer (SITC 2018) - O24 - A Phase 1, Open-Label, Dose Escalation Study of Enoblituzumab in Combination with Pembrolizumab in Patients with Select Solid Tumors. J Immunotherapy Cancer 2018;6:115.

65. Goswami CP, Nakshatri H. PROGgeneV2: enhancements on the existing database. BMC Cancer 2014;14:970.

66. Long L, Zhang X, Chen F, et al. The promising immune checkpoint LAG-3: from tumor microenvironment to cancer immunotherapy. Genes Cancer 2018;9:176-89.

67. Marcq E, Siozopoulou V, De Waele J, et al. Prognostic and predictive aspects of the tumor immune microenvironment and immune checkpoints in malignant pleural mesothelioma. Oncoimmunology 2016;6:e1261241.

68. Marcq E, Waele J, Audenaerde JV, et al. Abundant expression of TIM-3, LAG-3, PD-1 and PD-L1 as immunotherapy checkpoint targets in effusions of mesothelioma patients. Oncotarget 2017;8:89722-35.

69. Salaroglio IC, Kopecka J, Napoli F, et al. Potential Diagnostic and Prognostic Role of Microenvironment in Malignant Pleural Mesothelioma. J Thorac Oncol 2019;14:1458-71.

70. Chee J, Watson MW, Chopra A, et al. Tumour associated lymphocytes in the pleural effusions of patients with mesothelioma express high levels of inhibitory receptors. BMC Res Notes 2018;11:864.

71. Sheng CC, Han FY. Immunoregulation effects of TIM-3 on tumors. Neoplasma 2019;66:167-75.

72. Awad MM, Jones RE, Liu H, et al. Cytotoxic T Cells in PD-L1-Positive Malignant Pleural Mesotheliomas Are Counterbalanced by Distinct Immunosuppressive Factors. 
Cancer Immunol Res 2016;4:1038-48.

73. Sottile R, Tannazi M, Johansson MH, et al. NK- and T-cell subsets in malignant mesothelioma patients: Baseline pattern and changes in the context of anti-CTLA-4 therapy. Int J Cancer 2019;145:2238-48.

74. Fear VS, Tilsed C, Chee J, et al. Combination immune checkpoint blockade as an effective therapy for mesothelioma. Oncoimmunology 2018;7:e1494111.

75. Stone GW, Barzee S, Snarsky V, et al. Regression of established AB1 murine mesothelioma induced by peritumoral injections of $\mathrm{CpG}$ oligodeoxynucleotide either alone or in combination with poly(I:C) and CD40 ligand plasmid DNA. J Thorac Oncol 2009;4:802-8.

76. Lawler SE, Speranza MC, Cho CF, et al. Oncolytic Viruses in Cancer Treatment: A Review. JAMA Oncol 2017;3:841-9.

77. Harrington K, Freeman DJ, Kelly B, et al. Optimizing oncolytic virotherapy in cancer treatment. Nat Rev Drug Discov 2019;18:689-706.

78. Di Somma S, Iannuzzi CA, Passaro C, et al. The Oncolytic Virus d1922-947 Triggers Immunogenic Cell Death in Mesothelioma and Reduces Xenograft Growth. Front Oncol 2019;9:564.

79. Harada A, Uchino J, Harada T, et al. Vascular endothelial growth factor promoter-based conditionally replicative adenoviruses effectively suppress growth of malignant pleural mesothelioma. Cancer Sci 2017;108:116-23.

80. Achard C, Boisgerault N, Delaunay T, et al. Sensitivity of human pleural mesothelioma to oncolytic measles virus depends on defects of the type I interferon response. Oncotarget 2015;6:44892-904.

81. Takagi-Kimura M, Yamano T, Tamamoto A, et al. Enhanced antitumor efficacy of fiber-modified, midkine promoter-regulated oncolytic adenovirus in human malignant mesothelioma. Cancer Sci 2013;104:1433-9.

82. Belin LJ, Ady JW, Lewis C, et al. An oncolytic vaccinia virus expressing the human sodium iodine symporter prolongs survival and facilitates SPECT/CT imaging in an orthotopic model of malignant pleural mesothelioma. Surgery 2013;154:486-95.

83. Morodomi Y, Yano T, Kinoh H, et al. BioKnife, a uPA activity-dependent oncolytic Sendai virus, eliminates pleural spread of malignant mesothelioma via simultaneous stimulation of uPA expression. Mol Ther 2012;20:769-77.

84. Silberhumer GR, Brader P, Wong J, et al. Genetically engineered oncolytic Newcastle disease virus effectively induces sustained remission of malignant pleural mesothelioma. Mol Cancer Ther 2010;9:2761-9.
85. Kubo S, Kawasaki Y, Yamaoka N, et al. Complete regression of human malignant mesothelioma xenografts following local injection of midkine promoter-driven oncolytic adenovirus. J Gene Med 2010;12:681-92.

86. Kelly KJ, Woo Y, Brader P, et al. Novel oncolytic agent GLV-1h68 is effective against malignant pleural mesothelioma. Hum Gene Ther 2008;19:774-82.

87. Lambright ES, Caparrelli DJ, Abbas AE, et al. Oncolytic therapy using a mutant type- 1 herpes simplex virus and the role of the immune system. Ann Thorac Surg 1999;68:1756-60; discussion 1761-2.

88. Kucharczuk JC, Randazzo B, Chang MY, et al. Use of a "replication-restricted" herpes virus to treat experimental human malignant mesothelioma. Cancer Res 1997;57:466-71.

89. Sterman DH, Treat J, Litzky LA, et al. Adenovirusmediated herpes simplex virus thymidine kinase/ganciclovir gene therapy in patients with localized malignancy: results of a phase I clinical trial in malignant mesothelioma. Hum Gene Ther 1998;9:1083-92.

90. Sterman DH, Recio A, Vachani A, et al. Long-term follow-up of patients with malignant pleural mesothelioma receiving high-dose adenovirus herpes simplex thymidine kinase/ganciclovir suicide gene therapy. Clin Cancer Res 2005;11:7444-53.

91. Danson S, Woll P, Edwards J, et al. 366PD - Oncolytic herpesvirus therapy for mesothelioma: A phase I/IIa trial of intrapleural administration of HSV1716 (NCT01721018). Ann Oncol 2017;28:v122-41.

92. Kuryk L, Haavisto E, Garofalo M, et al. Synergistic anti-tumor efficacy of immunogenic adenovirus ONCOS-102 (Ad5/3-D24-GM-CSF) and standard of care chemotherapy in preclinical mesothelioma model. Int $\mathrm{J}$ Cancer 2016;139:1883-93.

93. Targovax announces early signal of efficacy in ONCOS-102 trial in mesothelioma. Available online: https:// wwwtargovaxcom/en/targovax-announces-early-signal-ofefficacy-in-oncos-102-trial-in-mesothelioma/. 2018.

94. Targovax announces completed enrollment of ONCOS-102 trial in mesothelioma. Available online: https://wwwtargovaxcom/en/targovax-announcescompleted-enrollment-of-oncos-102-trial-inmesothelioma/. 2019.

95. Ranki T, Pesonen S, Hemminki A, et al. Phase I study with ONCOS-102 for the treatment of solid tumors - an evaluation of clinical response and exploratory analyses of immune markers. J Immunother Cancer 2016;4:17.

96. Niemann J, Woller N, Brooks J, et al. Molecular 
retargeting of antibodies converts immune defense against oncolytic viruses into cancer immunotherapy. Nat Commun 2019;10:3236.

97. Thayaparan T, Petrovic RM, Achkova DY, et al. CAR T-cell immunotherapy of MET-expressing malignant mesothelioma. Oncoimmunology 2017;6:e1363137.

98. Klampatsa A, Haas AR, Moon EK, et al. Chimeric Antigen Receptor (CAR) T Cell Therapy for Malignant Pleural Mesothelioma (MPM). Cancers (Basel) 2017. doi: 10.3390/ cancers9090115.

99. Moon EK, Carpenito C, Sun J, et al. Expression of a functional CCR2 receptor enhances tumor localization and tumor eradication by retargeted human $\mathrm{T}$ cells expressing a mesothelin-specific chimeric antibody receptor. Clin Cancer Res 2011;17:4719-30.

100.Adusumilli PS, Cherkassky L, Villena-Vargas J, et al. Regional delivery of mesothelin-targeted CAR T cell therapy generates potent and long-lasting CD4-dependent tumor immunity. Sci Transl Med 2014;6:261ra151.

101. Schuberth PC, Hagedorn C, Jensen SM, et al. Treatment of malignant pleural mesothelioma by fibroblast activation protein-specific re-directed T cells. J Transl Med 2013;11:187.

102.Klampatsa A, Achkova DY, Davies DM, et al. Intracavitary 'T4 immunotherapy' of malignant mesothelioma using pan-ErbB re-targeted CAR T-cells. Cancer Lett 2017;393:52-9.

103. Kiesgen S, Chicaybam L, Chintala NK, et al. Chimeric Antigen Receptor (CAR) T-Cell Therapy for Thoracic Malignancies. J Thorac Oncol 2018;13:16-26.

104. Beatty GL, Haas AR, Maus MV, et al. Mesothelin-specific chimeric antigen receptor $\mathrm{mRNA}$-engineered $\mathrm{T}$ cells induce anti-tumor activity in solid malignancies. Cancer Immunol Res 2014;2:112-20.

105. Adusumilli PS, Zauderer MG, Rusch VW, et al. Abstract CT036: A phase I clinical trial of malignant pleural disease treated with regionally delivered autologous mesothelintargeted CAR T cells: Safety and efficacy. Cancer Res 2019;79:abstr CT036.

106. Wherry EJ. T cell exhaustion. Nature Immunology 2011;12:492.

107. Cherkassky L, Morello A, Villena-Vargas J, et al. Human CAR T cells with cell-intrinsic PD-1 checkpoint blockade resist tumor-mediated inhibition. J Clin Invest 2016;126:3130-44.

108. Cornelissen R, Hegmans JP, Maat AP, et al. Extended Tumor Control after Dendritic Cell Vaccination with Low-Dose Cyclophosphamide as Adjuvant Treatment in Patients with Malignant Pleural Mesothelioma. Am J

Respir Crit Care Med 2016;193:1023-31.

109. Gregoire M. Dendritic cell vaccination as a treatment modality for mesothelioma. Expert Rev Respir Med 2010;4:311-4.

110.Hegmans JP, Hemmes A, Aerts JG, et al. Immunotherapy of murine malignant mesothelioma using tumor lysatepulsed dendritic cells. Am J Respir Crit Care Med 2005;171:1168-77.

111.Hegmans JP, Veltman JD, Lambers ME, et al. Consolidative dendritic cell-based immunotherapy elicits cytotoxicity against malignant mesothelioma. Am J Respir Crit Care Med 2010;181:1383-90.

112. Peikert T, Sterman DH. Harnessing the Power of the Host: Improving Dendritic Cell Vaccines for Malignant Pleural Mesothelioma. Am J Respir Crit Care Med 2016;193:943-5.

113. Aerts JG, de Goeje PL, Cornelissen R, et al. Autologous Dendritic Cells Pulsed with Allogeneic Tumor Cell Lysate in Mesothelioma: From Mouse to Human. Clin Cancer Res 2018;24:766-76.

114. Belderbos RA, Baas P, Berardi R, et al. A multicenter, randomized, phase II/III study of dendritic cells loaded with allogeneic tumor cell lysate (MesoPher) in subjects with mesothelioma as maintenance therapy after chemotherapy: DENdritic cell Immunotherapy for Mesothelioma (DENIM) trial. Transl Lung Cancer Res 2019;8:280-5.

115. Yang CH. EGFR tyrosine kinase inhibitors for the treatment of NSCLC in East Asia: present and future. Lung Cancer 2008;60 Suppl 2:S23-30.

116. Yang J, He X, Lv Q, et al. Management of Adverse Events in Cancer Patients Treated With PD-1/PD-L1 Blockade: Focus on Asian Populations. Front Pharmacol 2019;10:726.

117. Frelaut M, Le Tourneau C, Borcoman E. Hyperprogression under Immunotherapy. Int J Mol Sci 2019;20:2674.

118. Fuentes-Antrás J, Provencio M, Diaz-Rubio E. Hyperprogression as a distinct outcome after immunotherapy. Cancer Treat Rev 2018;70:16-21.

119. Popat S. Hyperprogression with immunotherapy: Is it real? Cancer 2019;125:1218-20.

120. Bonaventura P, Shekarian T, Alcazer V, et al. Cold Tumors: A Therapeutic Challenge for Immunotherapy. Front Immunol 2019;10:168.

121. Chiappinelli KB, Zahnow CA, Ahuja N, et al. Combining Epigenetic and Immunotherapy to Combat Cancer. 
Cancer Res 2016;76:1683-9.

122. Tsai HC, Li H, Van Neste L, et al. Transient low doses of DNA-demethylating agents exert durable antitumor effects on hematological and epithelial tumor cells. Cancer Cell 2012;21:430-46.

123. Woods DM, Sodre AL, Villagra A, et al. HDAC Inhibition Upregulates PD-1 Ligands in Melanoma and Augments Immunotherapy with PD-1 Blockade. Cancer Immunol Res 2015;3:1375-85.

124. Briere D, Sudhakar N, Woods DM, et al. The class I/IV HDAC inhibitor mocetinostat increases tumor antigen presentation, decreases immune suppressive cell types and augments checkpoint inhibitor therapy. Cancer Immunol Immunother 2018;67:381-92.

125. Gray JE, Saltos AN, Tanvetyanon T, et al. Phase 1/1b study of pembrolizumab plus vorinostat in advanced/ metastatic non-small cell lung cancer. Clin Cancer Res 2019;25:6623-32.

126. Entinostat Helps Thwart Immunotherapy Resistance. Cancer Discov 2019;9:685-6.

127.Johnson ML, Adjei AA, Ramalingam SS, et al. Preliminary results of ENCORE 601, a phase 1b/2, open-label study of entinostat (ENT) in combination with pembrolizumab (PEMBRO) in patients with non-small cell lung cancer (NSCLC). J Clin Oncol 2016;34:abstr e20659.

128. Stone ML, Chiappinelli KB, Li H, et al. Epigenetic therapy activates type I interferon signaling in murine ovarian cancer to reduce immunosuppression and tumor burden. Proc Natl Acad Sci U S A 2017;114:E10981-90. 129. Topper MJ, Vaz M, Chiappinelli KB, et al. Epigenetic

Cite this article as: Gray SG, Mutti L. Immunotherapy for mesothelioma: a critical review of current clinical trials and future perspectives. Transl Lung Cancer Res 2020;9(Suppl 1):S100-S119. doi: 10.21037/tlcr.2019.11.23
Therapy Ties MYC Depletion to Reversing Immune Evasion and Treating Lung Cancer. Cell 2017;171:1284300.e21.

130.Adeegbe DO, Liu Y, Lizotte PH, et al. Synergistic Immunostimulatory Effects and Therapeutic Benefit of Combined Histone Deacetylase and Bromodomain Inhibition in Non-Small Cell Lung Cancer. Cancer Discov 2017;7:852-67.

131. Krug LM, Kindler HL, Calvert H, et al. Vorinostat in patients with advanced malignant pleural mesothelioma who have progressed on previous chemotherapy (VANTAGE-014): a phase 3, double-blind, randomised, placebo-controlled trial. Lancet Oncol 2015;16:447-56.

132. Bensaid D, Blondy T, Deshayes S, et al. Assessment of new HDAC inhibitors for immunotherapy of malignant pleural mesothelioma. Clin Epigenetics 2018;10:79.

133. Tallón de Lara P, Cecconi V, Hiltbrunner S, et al. Gemcitabine Synergizes with Immune Checkpoint Inhibitors and Overcomes Resistance in a Preclinical Model and Mesothelioma Patients. Clin Cancer Res 2018;24:6345-54.

134. Gray SG, Baird AM, O'Kelly F, et al. Gemcitabine reactivates epigenetically silenced genes and functions as a DNA methyltransferase inhibitor. Int J Mol Med 2012;30:1505-11.

135.Magnuson AM, Kiner E, Ergun A, et al. Identification and validation of a tumor-infiltrating Treg transcriptional signature conserved across species and tumor types. Proc Natl Acad Sci U S A 2018;115:E10672-81. 